\title{
Estimation of Genetic Variability and Divergence for Fruit Yield and Quality Traits in Cucumber (Cucumis Sativus L.) in North-Western Himalays
}

\author{
Sandeep Kumar ${ }^{1}$, Dharminder Kumar ${ }^{1, *}$, Ramesh Kumar ${ }^{1}$, KS Thakur ${ }^{1}$, Balbir Singh Dogra ${ }^{2}$ \\ ${ }^{1}$ Department of Vegetable Science, Dr. YS Parmar University of Horticulture and Forestry, Nauni, Solan-173 230, HP, India \\ ${ }^{2}$ Regional Horticulture and Forestry Research Station, Bhota, Hamirpur (HP), Dr. YS Parmar University of Horticulture and Forestry, \\ Nauni, Solan-173 230, HP, India \\ * Corresponding Author: dharmruder@gmail.com
}

Copyright (C) 2013 Horizon Research Publishing All rights reserved.

\begin{abstract}
Thirty diverse genotypes of cucumber collected from different indigenous sources were planted in randomized complete block design, during rainy season of 2009 and were assessed to know the nature and magnitude of variability and genetic divergence for different horticultural traits. The genotype LC-1 gave maximum mean value for fruit weight and yield per plot. High phenotypic coefficient of variability (PCV), genotypic coefficient of variability (GCV) and heritability estimates coupled with high genetic gain were observed for seed vigour index-I and yield per plot, indicated the existence of wide range of variations and offers better scope for improvement through selection. The genotypes were grouped into 4 clusters and the highest (6.168) inter cluster distance was recorded between cluster-II and III. The diverse genotypes characterized by maximum inter cluster distance will differ in phenotypic performance and therefore, selection of divergent parents should be based on these cluster distances to obtain favourable hybrids and transgressive segregants in cucumber.
\end{abstract}

Keywords Cucumber, Genetic Variability, Divergence, Heritability and Mahalanobis $\mathrm{D}^{2}$ statistics

\section{Introduction}

Cucumber (Cucumis sativus L.) is one of the most important cucurbitaceous vegetable crops grown extensively in tropical and sub-tropical parts of the country. It is considered as 4th most important vegetable crop after tomato, cabbage and onion (Tatlioglu, 1993). Cucumber is a thermophilic and frost susceptible crop species, growing best at a temperature above $20^{\circ} \mathrm{C}$. It is grown for its tender fruits, which are consumed either raw as salad, cooked as vegetable or as pickling cucumber in its immature stage. It is a rich source of vitamin $\mathrm{B}$ and $\mathrm{C}$, carbohydrates, $\mathrm{Ca}$ and $\mathrm{P}$
(Yawalkar, 1985).

In spite of being native of Indian sub-continent and endowed with enormous variability and genetic divergence, cucumber remains underutilized in terms of its economic potential and unexploited from breeding point of view. So, there is a great need of screening cucumber germplasm to select elite genotypes with improved quality and higher yield for direct selection or using as a parent in hybridization programme.

Planning and execution of breeding programme for improvement of quantitative attributes depends, to a great extent, upon magnitude of genetic variability. The genotypic and phenotypic coefficients of variability are helpful in exploring the nature of variability in the breeding populations, whereas estimates of heritability provides index of transmissibility of characters. Hence, on the basis of these parameters, suitable selection strategy can be formulated for higher yield in cucumber. Information on genetic diversity is used to identify the promising diverse genotypes, which may be used in further breeding programme.

Therefore, keeping in view the importance of above facts in mind, the present study has been undertaken to estimate the extent of variability, heritability, coefficients of variation, genetic advance and genetic divergence in thirty diverse genotypes of cucumber.

\section{Materials and Methods}

\subsection{Experimental Site and Environment}

The present investigations were carried out at Research Farm of the Department of Vegetable Science, Dr. Y.S. Parmar University of Horticulture and Forestry, Nauni, Solan in the state of Himachal Pradesh, India during rainy season of 2009 . This location is at $30^{\circ} 50^{\prime} \mathrm{N}$ latitude and $77^{\circ} 11^{\prime} 30^{\prime \prime}$ E longitude and is $1260 \mathrm{~m}$ above mean sea level and represents the mid-hill zone of Himachal Pradesh. The 
total rainfall during growing season was $381.90 \mathrm{~mm}$, most of which was received in July month viz. $187.30 \mathrm{~mm}$. Maximum mean temperature varied from $27.2^{\circ} \mathrm{C}$ to $33.3^{\circ} \mathrm{C}$ and minimum from $15.9^{\circ} \mathrm{C}$ to $19.5^{\circ} \mathrm{C}$. Maximum relative humidity recorded was 76.0 per cent and minimum was 44.6 per cent during the growing season.

Table 1. List of cucumber genotypes studied along with their sources

\begin{tabular}{|l|l|}
\hline \multicolumn{1}{|c|}{ Genotype } & \multicolumn{1}{c|}{ Source } \\
\hline $\begin{array}{l}\text { LC-1, LC-2, LC-3, LC-4, } \\
\text { LC-5, LC-6 }\end{array}$ & Hamirpur, Himachal Pradesh, India \\
\hline LC-7, LC-8 & Bilaspur, Himachal Pradesh, India \\
\hline LC-9, LC-10, LC-11, LC-12 & Kangra, Himachal Pradesh, India \\
\hline LC-13, LC-14, LC-15 & Mandi, Himachal Pradesh, India \\
\hline LC-16, LC-17 & Shimla, Himachal Pradesh, India \\
\hline LC-18, LC-19, LC-20 & Kullu, Himachal Pradesh, India \\
\hline LC-21, LC-22 & Chamba, Himachal Pradesh, India \\
\hline LC-23, LC-24, LC-25 & Solan, Himachal Pradesh, India \\
\hline LC-26, LC-27 & Una, Himachal Pradesh, India \\
\hline LC-28 & Jammu, Jammu \& Kashmir, India \\
\hline K-75*, K-90* & $\begin{array}{l}\text { UHF, Nauni, Solan, Himachal } \\
\text { Pradesh, India }\end{array}$ \\
\hline
\end{tabular}

${ }^{*}$ Control

\subsection{Experimental Material, Layout and Observations}

The experimental material consisted of diverse group of 30 genotypes of cucumber, including check cultivars i.e., K-75 and K-90 (Table 1) collected from different regions of North-Western Himalays having tremendous genetic diversity of cucumber. The experiment was laid out in randomized block design with three replications of each genotype. The experimental field was disked and levelled. About $10 \mathrm{Mt} \mathrm{ha}^{-1}$ of well decomposed farm yard manure was mixed in the soil at the time of field preparation. The recommended fertilizer dose of $\mathrm{N}: \mathrm{P}_{2} \mathrm{O}_{5}: \mathrm{K}_{2} \mathrm{O}$ was applied at the time of sowing at the rate of 400,315 and $100 \mathrm{Kg} \mathrm{ha}^{-1}$ as calcium ammonium nitrate, single- superphosphate, and muriate of potash, respectively. Seeds were directly sown in the field in the month of May, 2009. Three to four seeds per basin were sown at a spacing of $100 \times 75 \mathrm{~cm}$ in a plot having size of $3.0 \times 2.25 \mathrm{~m}^{2}$, accommodating 9 plants per plot. After the emergence of seedlings, only one healthy seedling per hill was retained. Standard cultural practices recommended in the Package of Practices for Vegetable Crops, were followed to ensure a healthy crop stand (Anonymous, 2009). The observations were recorded on node number bearing first female flower, days to marketable maturity, number of marketable fruits per plant, harvest duration, fruit length, fruit breadth, average fruit weight, total soluble solids, yield per plot, seed germination, seed vigour index-I and II and severity of powdery mildew, anthracnose and angular leaf spot from five randomly selected plants in each replication for all characters except for fruit characters for which observations were recorded on ten randomly selected fruits per replication. Colour of fruits was observed visually after harvesting with the help of colour chart of Royal Horticultural Society, London. Seed germination of each genotype was tested in accordance with ISTA (Anonymous, 1985) and seed vigour index-I and II were calculated as per the formula given by Abdul-Baki and Anderson (1973). The disease severity of anthracnose and angular leaf spot was recorded on 0-5 scale as suggested by Bhat (2007) and disease severity for powdery mildew was recorded by adopting the scale given by Ransom et al. (1991).

\subsection{Statistical Analysis}

The data were subjected to analysis of variance as per procedure described by Gomez and Gomez (1983). The genotypic and phenotypic coefficients of variation were calculated as per formulae given by Burton and De-Vane (1953). Heritability and genetic advance were calculated according to Allard (1960) and genetic gain was estimated as per the method given by Johanson et al. (1955). Multivariate analysis was done utilizing Mahalanobis $\mathrm{D}^{2}$ statistics and genotypes were grouped into four different clusters following Tochers method as described by Rao (1952).

\section{Results and Discussion}

\subsection{Variability studies}

\subsubsection{Analysis of Variance}

Genetic variability is the basic need for a plant breeder to initiate any breeding programme. Genetic improvement can be brought about by manipulating the genetic makeup of the plant for desirable characters or to remove the undesirable genes which retard, or inhibit, certain pathways. Analysis of variance (Table 2) indicated significant differences among the genotypes for all the characters under study. These differences indicated the presence of variability in the available germplasm and offers opportunity for improvement in yield and quality traits of cucumber. 
Table 2. Analysis of variance for different horticultural traits in cucumber

\begin{tabular}{|c|c|c|c|c|}
\hline \multirow{2}{*}{ Character } & \multicolumn{4}{|c|}{ Source of variation } \\
\hline & Genotype $(29)^{a}$ & Replication (2) & Error (58) & Total (89) \\
\hline Node number bearing first female flower & $23.61 *$ & 114.00 & 5.29 & 142.90 \\
\hline Days to marketable maturity & $119.11^{*}$ & 289.43 & 15.36 & 423.90 \\
\hline Number of marketable fruits / plant & $3.70 *$ & 3.52 & 0.29 & 7.51 \\
\hline Harvest duration (days) & $63.14 *$ & 249.87 & 10.75 & 323.76 \\
\hline Fruit length $(\mathrm{cm})$ & $26.07 *$ & 17.90 & 0.97 & 44.94 \\
\hline Fruit breadth $(\mathrm{cm})$ & $1.68^{*}$ & 0.31 & 0.08 & 2.07 \\
\hline Average fruit weight (g) & $12518.49 *$ & 11884.63 & 649.26 & 25052.38 \\
\hline $\operatorname{TSS}\left({ }^{0} \mathrm{~B}\right)$ & $0.95^{*}$ & 2.24 & 0.11 & 3.30 \\
\hline Seed germination $(\%)$ & $203.06^{*}$ & 161.47 & 12.46 & 376.99 \\
\hline Seed vigor index-I & $664805.62 *$ & 2229599.67 & 96125.43 & 2990530.70 \\
\hline Seed vigor index-II & $684473.69^{*}$ & 2007898.64 & 96649.04 & 2789021.40 \\
\hline Severity of powdery mildew (\%) & $95.20^{*}$ & 424.36 & 19.13 & 538.69 \\
\hline Severity of anthracnose (\%) & $62.95^{*}$ & 422.92 & 18.61 & 504.48 \\
\hline Severity of angular leaf spot (\%) & $33.43 *$ & 231.96 & 10.17 & 275.56 \\
\hline Yield/plot (kg) & $86.71^{*}$ & 5.94 & 1.51 & 94.16 \\
\hline Yield/hectare (q) [converted] & $9325.70 *$ & 639.59 & 162.57 & 10127.86 \\
\hline
\end{tabular}

*Significant at $5 \%$ level of significance

${ }^{a}$ Values in the parenthesis are degree of freedom

\subsubsection{Mean Performance of Genotypes}

Genetic variability is the basic need for a plant breeder to initiate any breeding programme. Among the horticultural traits, comparatively wide range was observed for node number bearing first female flower (3.53-13.53) and days to marketable maturity (55.67-78.33), which determine the earliness of a variety (Table 3 ). Fruit length, breadth and weight are the major yield contributing traits, wide variations were observed with respect to these traits (8.11-22.76 cm, 3.08-7.18 cm, 95.00-430.00 g, respectively). Tremendous variations with respect to number of marketable fruits per plant (5.01 - 8.57), harvest duration (14.00 - 28.67 days) and yield per plot $(4.37-27.31 \mathrm{~kg})$ were obtained. The genotype LC-1 was found highest in yield $(27.31 \mathrm{~kg} / \mathrm{ha})$ among all and four other genotypes namely LC-2, LC-3, LC-15 and LC-28 gave higher yield than both the checks. Wide variations with respect to various horticultural characters were also reported by Singh et al. (2002), Das et al. (2003), Verma (2003), Kumar (2006), Munshi et al. (2007), Kumar et al. (2008), Hanchinamani et al. (2008) and Yogesh et al. (2009) in cucumber.

All the genotypes under study also showed wide variations for fruit colour (green, light green, dark green and white) and total soluble solids $(2.03-4.07 \mathrm{OB})$, which decides the consumer's preference. Majority of the genotypes including both the check cultivars had light green coloured fruits, which are in general preferred by consumers. Higher TSS was recorded in LC-28 (4.07 0B) and six other genotypes namely LC-2, LC-3, LC-15, LC-19, LC-25 and LC-26 performed better over both the checks for total soluble solids. Similar results have also been reported by Verma (2003) and Kumar (2006) for these characters.

For seed characters viz. seed germination (61.00-87.67\%), seed vigor index-I (438.33-1930.00) and seed vigor index-II (1642.28-3167.28), a wide variation was observed. Maximum seed germination was recorded in LC-6 and LC-20 (87.67), respectively, while LC-3 performed better for seed vigor index-I and II (1930.00 and 3167.28, respectively). Similar results were also reported by Hamid et al. (2002) for seed germination and by Nerson (2007) for seedling vigour.

All the genotypes studied, respond differently to the attack of different diseases viz. powdery mildew (8.50-29.40\%), anthracnose $(7.70-26.20 \%)$ and angular leaf spot (6.50-18.30\%). Minimum severity of powdery mildew, anthracnose and angular leaf spot was recorded in LC-21 (8.50 \%), LC-15 (7.70 \%) and LC-22 (6.50\%), respectively. Hence, these genotypes can be used as resistant sources in further breeding programmes. These findings are in agreement with Webner and Shetty (2000) and Cohen et al. (1995). These wide variations in the genotypes for different characters would help in selecting the best genotypes from existing collections. 
Table 3. Mean performance of cucumber genotypes for different horticultural traits

\begin{tabular}{|c|c|c|c|c|c|c|c|c|c|c|c|c|c|c|c|c|}
\hline $\begin{array}{c}\text { Genotyp } \\
\text { e }\end{array}$ & NNBFF & DMM & NMF & HD & FL & FB & AFW & FC & TSS & $\mathrm{SG}^{*}$ & SVI- I & SVI-II & $\mathrm{SPM}^{* *}$ & $\mathrm{SA}^{* *}$ & SALS** & YPP \\
\hline LC-1 & 7.47 & 75.67 & 7.08 & 25.33 & 21.71 & 6.58 & 430.00 & $\begin{array}{l}\text { LG (G } \\
141 C)\end{array}$ & 2.57 & $\begin{array}{c}72.33 \\
(58.25) \\
\end{array}$ & 1345.00 & 2438.67 & $\begin{array}{l}16.50 \\
(3.99) \\
\end{array}$ & $\begin{array}{l}10.30 \\
(3.02) \\
\end{array}$ & $\begin{array}{c}7.35 \\
(2.59) \\
\end{array}$ & 27.31 \\
\hline LC-2 & 6.23 & 66.33 & 8.06 & 26.33 & 15.76 & 5.73 & 331.67 & $\begin{array}{l}\text { LG (G } \\
134 \mathrm{D})\end{array}$ & 3.77 & $\begin{array}{c}76.33 \\
(60.89) \\
\end{array}$ & 1729.00 & 2968.97 & $\begin{array}{l}19.70 \\
(4.41)\end{array}$ & $\begin{array}{c}8.40 \\
(2.78)\end{array}$ & $\begin{array}{c}8.55 \\
(2.88) \\
\end{array}$ & 23.91 \\
\hline LC-3 & 6.00 & 65.33 & 8.07 & 24.00 & 16.56 & 5.37 & 303.33 & $\begin{array}{l}\text { LG (G } \\
130 \mathrm{C})\end{array}$ & 3.53 & $\begin{array}{l}79.33 \\
(63.06) \\
\end{array}$ & 1930.00 & 3167.28 & $\begin{array}{l}14.70 \\
(3.77)\end{array}$ & $\begin{array}{l}10.50 \\
(3.09)\end{array}$ & $\begin{array}{l}11.60 \\
(3.35) \\
\end{array}$ & 21.96 \\
\hline LC-4 & 11.00 & 73.67 & 6.04 & 17.67 & 11.15 & 4.48 & 143.33 & $\begin{array}{l}\text { LG (G } \\
136 \mathrm{C}) \\
\end{array}$ & 2.07 & $\begin{array}{c}62.67 \\
(52.35) \\
\end{array}$ & 765.33 & 1665.67 & $\begin{array}{l}21.30 \\
(4.59) \\
\end{array}$ & $\begin{array}{l}15.30 \\
(3.87) \\
\end{array}$ & $\begin{array}{l}12.70 \\
(3.54) \\
\end{array}$ & 7.67 \\
\hline LC-5 & 10.87 & 74.67 & 6.57 & 19.33 & 12.43 & 5.27 & 248.33 & $\begin{array}{l}\text { LG (G } \\
141 C)\end{array}$ & 2.77 & $\begin{array}{c}70.67 \\
(57.20) \\
\end{array}$ & 666.33 & 1918.67 & $\begin{array}{l}18.00 \\
(4.22)\end{array}$ & $\begin{array}{l}18.80 \\
(4.31)\end{array}$ & $\begin{array}{c}8.20 \\
(2.82) \\
\end{array}$ & 14.68 \\
\hline LC-6 & 9.50 & 74.33 & 6.79 & 20.67 & 12.53 & 5.18 & 246.67 & $\begin{array}{c}G(G \\
141 B) \\
\end{array}$ & 2.77 & $\begin{array}{c}87.67 \\
(69.67) \\
\end{array}$ & 1570.00 & 2873.67 & $\begin{array}{l}21.10 \\
(4.55)\end{array}$ & $\begin{array}{c}9.40 \\
(2.91)\end{array}$ & $\begin{array}{l}13.30 \\
(3.60) \\
\end{array}$ & 14.81 \\
\hline LC-7 & 10.47 & 77.67 & 5.60 & 14.33 & 12.48 & 5.00 & 236.67 & $\begin{array}{l}\text { LG (G } \\
143 B)\end{array}$ & 2.10 & $\begin{array}{c}61.67(51 \\
.75) \\
\end{array}$ & 484.33 & 1790.02 & $\begin{array}{l}22.30 \\
(4.69) \\
\end{array}$ & $\begin{array}{l}16.45 \\
(4.00) \\
\end{array}$ & $\begin{array}{l}13.60 \\
(3.66) \\
\end{array}$ & 11.68 \\
\hline LC-8 & 13.07 & 78.33 & 5.68 & 16.00 & 15.47 & 7.18 & 343.33 & $\begin{array}{l}\mathrm{W}(\mathrm{W} \\
155 \mathrm{~A}) \\
\end{array}$ & 2.17 & $\begin{array}{c}62.00 \\
(51.95) \\
\end{array}$ & 438.33 & 1652.00 & $\begin{array}{l}24.50 \\
(4.93) \\
\end{array}$ & $\begin{array}{l}11.80 \\
(3.38) \\
\end{array}$ & $\begin{array}{l}15.40 \\
(3.91) \\
\end{array}$ & 17.54 \\
\hline LC-9 & 11.27 & 75.33 & 6.40 & 17.33 & 15.59 & 5.46 & 290.00 & $\begin{array}{l}\text { LG (G } \\
141 C) \\
\end{array}$ & 2.43 & $\begin{array}{c}70.00 \\
(56.86) \\
\end{array}$ & 1438.00 & 2801.67 & $\begin{array}{l}29.40 \\
(5.40) \\
\end{array}$ & $\begin{array}{l}12.50 \\
(3.44) \\
\end{array}$ & $\begin{array}{c}8.30 \\
(2.80) \\
\end{array}$ & 16.63 \\
\hline LC-10 & 11.50 & 76.67 & 5.40 & 14.33 & 14.27 & 5.18 & 251.67 & $\begin{array}{c}G(G \\
135 C)\end{array}$ & 2.07 & $\begin{array}{c}61.33 \\
(51.54) \\
\end{array}$ & 608.00 & 1880.48 & $\begin{array}{l}23.30 \\
(4.79)\end{array}$ & $\begin{array}{l}13.80 \\
(3.64)\end{array}$ & $\begin{array}{l}15.50 \\
(3.91) \\
\end{array}$ & 12.18 \\
\hline LC-11 & 8.67 & 74.33 & 5.09 & 14.00 & 13.31 & 3.08 & 146.67 & $\begin{array}{c}\mathrm{G}(\mathrm{G} \\
135 \mathrm{C})\end{array}$ & 2.43 & $\begin{array}{c}62.33 \\
(52.13) \\
\end{array}$ & 463.00 & 1642.28 & $\begin{array}{l}25.50 \\
(5.02)\end{array}$ & $\begin{array}{l}14.60 \\
(3.76)\end{array}$ & $\begin{array}{l}15.30 \\
(3.88)\end{array}$ & 6.66 \\
\hline LC-12 & 9.68 & 67.33 & 7.15 & 19.33 & 16.64 & 3.88 & 241.67 & $\begin{array}{c}\mathrm{G}(\mathrm{G} \\
141 \mathrm{~B})\end{array}$ & 2.83 & $\begin{array}{c}64.00 \\
(53.13) \\
\end{array}$ & 1166.67 & 2157.90 & $\begin{array}{l}17.70 \\
(4.16)\end{array}$ & $\begin{array}{l}25.30 \\
(5.01)\end{array}$ & $\begin{array}{c}7.10 \\
(2.57)\end{array}$ & 15.43 \\
\hline LC-13 & 11.20 & 75.33 & 5.17 & 14.67 & 8.11 & 4.81 & 95.00 & $\begin{array}{c}\mathrm{G}(\mathrm{G} \\
132 \mathrm{C}) \\
\end{array}$ & 2.03 & $\begin{array}{c}76.67 \\
(61.17) \\
\end{array}$ & 721.67 & 1967.93 & $\begin{array}{l}19.80 \\
(4.41) \\
\end{array}$ & $\begin{array}{l}17.10 \\
(4.08) \\
\end{array}$ & $\begin{array}{l}18.30 \\
(4.26) \\
\end{array}$ & 4.37 \\
\hline LC-14 & 4.87 & 61.33 & 7.12 & 21.33 & 13.12 & 4.98 & 243.33 & $\begin{array}{l}\text { LG (G } \\
141 C)\end{array}$ & 2.73 & $\begin{array}{c}66.33 \\
(54.53) \\
\end{array}$ & 601.00 & 1926.73 & $\begin{array}{l}14.40 \\
(3.76) \\
\end{array}$ & $\begin{array}{l}21.50 \\
(4.62) \\
\end{array}$ & $\begin{array}{c}7.20 \\
(2.63) \\
\end{array}$ & 15.61 \\
\hline LC-15 & 4.67 & 58.67 & 8.57 & 28.67 & 14.70 & 5.46 & 285.00 & $\begin{array}{c}G(G \\
141 B) \\
\end{array}$ & 3.20 & $\begin{array}{c}78.33 \\
(62.25) \\
\end{array}$ & 1736.67 & 2367.00 & $\begin{array}{l}12.30 \\
(3.44) \\
\end{array}$ & $\begin{array}{c}7.70 \\
(2.60) \\
\end{array}$ & $\begin{array}{l}13.60 \\
(3.67) \\
\end{array}$ & 21.91 \\
\hline LC-16 & 9.10 & 76.67 & 5.40 & 15.00 & 10.11 & 4.01 & 130.00 & $\begin{array}{l}\text { LG (G } \\
140 B)\end{array}$ & 2.33 & $\begin{array}{c}62.67 \\
(52.35) \\
\end{array}$ & 486.33 & 1932.89 & $\begin{array}{l}25.50 \\
(5.03) \\
\end{array}$ & $\begin{array}{l}20.80 \\
(4.54) \\
\end{array}$ & $\begin{array}{c}9.50 \\
(3.04) \\
\end{array}$ & 6.25 \\
\hline LC-17 & 13.53 & 78.33 & 5.01 & 14.67 & 11.59 & 5.05 & 235.00 & $\begin{array}{l}\text { LG (G } \\
142 B)\end{array}$ & 2.23 & $\begin{array}{c}62.33 \\
(52.13) \\
\end{array}$ & 624.67 & 1836.00 & $\begin{array}{l}24.60 \\
(4.93) \\
\end{array}$ & $\begin{array}{l}19.50 \\
(4.37) \\
\end{array}$ & $\begin{array}{l}10.50 \\
(3.19) \\
\end{array}$ & 10.52 \\
\hline LC-18 & 6.73 & 67.67 & 6.27 & 16.33 & 14.73 & 5.23 & 258.33 & $\begin{array}{l}\text { LG (G } \\
136 \mathrm{C})\end{array}$ & 2.70 & $\begin{array}{c}61.00 \\
(51.35)\end{array}$ & 458.67 & 1889.48 & $\begin{array}{l}28.80 \\
(5.34)\end{array}$ & $\begin{array}{l}14.40 \\
(3.73)\end{array}$ & $\begin{array}{l}13.50 \\
(3.64)\end{array}$ & 14.50 \\
\hline
\end{tabular}




\begin{tabular}{|c|c|c|c|c|c|c|c|c|c|c|c|c|c|c|c|c|}
\hline LC-19 & 5.20 & 65.67 & 7.01 & 17.33 & 14.08 & 4.73 & 241.67 & $\begin{array}{c}G(G \\
141 B)\end{array}$ & 3.37 & $\begin{array}{c}74.67 \\
(59.88) \\
\end{array}$ & 1166.67 & 2671.00 & $\begin{array}{l}20.50 \\
(4.49) \\
\end{array}$ & $\begin{array}{l}12.70 \\
(3.49) \\
\end{array}$ & $\begin{array}{c}9.50 \\
(3.03) \\
\end{array}$ & 15.17 \\
\hline LC-20 & 11.93 & 76.33 & 6.04 & 17.00 & 13.17 & 4.78 & 223.33 & $\begin{array}{l}\text { LG (G } \\
135 \mathrm{D})\end{array}$ & 2.33 & $\begin{array}{c}87.67 \\
(69.67) \\
\end{array}$ & 1360.00 & 2997.00 & $\begin{array}{l}21.60 \\
(4.61)\end{array}$ & $\begin{array}{l}15.20 \\
(3.84)\end{array}$ & $\begin{array}{l}13.90 \\
(3.70)\end{array}$ & 12.05 \\
\hline LC-21 & 8.33 & 74.67 & 8.02 & 26.67 & 13.14 & 5.23 & 266.67 & $\begin{array}{c}\text { LG } \\
\text { (G130D) }\end{array}$ & 3.07 & $\begin{array}{c}75.67 \\
(60.51) \\
\end{array}$ & 1400.00 & 2431.67 & $\begin{array}{c}8.50 \\
(2.68) \\
\end{array}$ & $\begin{array}{l}12.50 \\
(3.43) \\
\end{array}$ & $\begin{array}{l}13.60 \\
(3.65) \\
\end{array}$ & 19.11 \\
\hline LC-22 & 3.53 & 55.67 & 6.02 & 15.33 & 13.02 & 5.08 & 251.67 & $\begin{array}{c}\mathrm{G}(\mathrm{G} \\
134 \mathrm{~B})\end{array}$ & 2.43 & $\begin{array}{c}66.00 \\
(54.32) \\
\end{array}$ & 714.67 & 1976.33 & $\begin{array}{l}26.50 \\
(5.14) \\
\end{array}$ & $\begin{array}{l}21.10 \\
(4.59) \\
\end{array}$ & $\begin{array}{c}6.50 \\
(2.48) \\
\end{array}$ & 13.55 \\
\hline LC-23 & 12.47 & 75.67 & 5.11 & 14.67 & 11.68 & 4.98 & 228.33 & $\begin{array}{c}\mathrm{G}(\mathrm{G} \\
135 \mathrm{C}) \\
\end{array}$ & 2.47 & $\begin{array}{c}61.67 \\
(51.73) \\
\end{array}$ & 494.33 & 1655.39 & $\begin{array}{l}25.70 \\
(5.06) \\
\end{array}$ & $\begin{array}{l}16.80 \\
(4.08) \\
\end{array}$ & $\begin{array}{l}15.20 \\
(3.88) \\
\end{array}$ & 10.39 \\
\hline LC-24 & 10.70 & 74.67 & 5.97 & 16.33 & 13.65 & 5.00 & 265.00 & $\begin{array}{c}\mathrm{G}(\mathrm{G} \\
137 \mathrm{~A})\end{array}$ & 2.07 & $\begin{array}{c}65.67 \\
(54.13) \\
\end{array}$ & 612.33 & 1876.80 & $\begin{array}{l}21.60 \\
(4.64) \\
\end{array}$ & $\begin{array}{l}11.80 \\
(3.41) \\
\end{array}$ & $\begin{array}{l}12.30 \\
(3.48) \\
\end{array}$ & 14.14 \\
\hline LC-25 & 8.37 & 74.33 & 6.54 & 18.00 & 22.76 & 4.29 & 251.67 & $\begin{array}{c}\mathrm{G}(\mathrm{G} \\
135 \mathrm{C})\end{array}$ & 3.17 & $\begin{array}{c}69.33 \\
(56.37) \\
\end{array}$ & 586.33 & 1846.93 & $\begin{array}{l}13.50 \\
(3.65) \\
\end{array}$ & $\begin{array}{l}26.20 \\
(5.11) \\
\end{array}$ & $\begin{array}{l}11.20 \\
(3.31) \\
\end{array}$ & 14.67 \\
\hline LC-26 & 8.10 & 73.67 & 6.56 & 17.33 & 12.19 & 4.53 & 225.00 & $\begin{array}{l}\text { LG (G } \\
130 \mathrm{C})\end{array}$ & 3.77 & $\begin{array}{c}84.00 \\
(66.44) \\
\end{array}$ & 1733.67 & 2766.33 & $\begin{array}{c}9.50 \\
(3.01)\end{array}$ & $\begin{array}{l}15.90 \\
(3.96)\end{array}$ & $\begin{array}{l}17.80 \\
(4.20)\end{array}$ & 13.14 \\
\hline LC-27 & 4.23 & 60.33 & 8.38 & 21.67 & 14.28 & 4.35 & 236.33 & $\begin{array}{l}\mathrm{DG}(\mathrm{G} \\
136 \mathrm{~A}) \\
\end{array}$ & 2.80 & $\begin{array}{c}81.67 \\
(64.65) \\
\end{array}$ & 1340.00 & 2913.00 & $\begin{array}{l}11.50 \\
(3.37) \\
\end{array}$ & $\begin{array}{l}12.50 \\
(3.52) \\
\end{array}$ & $\begin{array}{l}12.40 \\
(3.50) \\
\end{array}$ & 17.77 \\
\hline LC-28 & 7.40 & 67.33 & 8.15 & 26.33 & 16.58 & 5.40 & 308.33 & $\begin{array}{c}G(G \\
141 B)\end{array}$ & 4.07 & $\begin{array}{c}73.67 \\
(59.12) \\
\end{array}$ & 1216.67 & 2562.00 & $\begin{array}{l}12.40 \\
(3.47)\end{array}$ & $\begin{array}{l}13.60 \\
(3.65)\end{array}$ & $\begin{array}{l}7.10 \\
(2.55)\end{array}$ & 22.58 \\
\hline K-75 & 6.16 & 66.67 & 7.91 & 24.67 & 13.23 & 4.91 & 261.67 & $\begin{array}{l}\text { LG (G } \\
141 C)\end{array}$ & 3.07 & $\begin{array}{c}72.67 \\
(58.53) \\
\end{array}$ & 1072.67 & 2433.33 & $\begin{array}{l}18.50 \\
(4.28) \\
\end{array}$ & $\begin{array}{l}14.20 \\
(3.74) \\
\end{array}$ & $\begin{array}{l}11.40 \\
(3.33)\end{array}$ & 18.56 \\
\hline K-90 & 6.60 & 73.33 & 8.02 & 26.00 & 15.85 & 5.25 & 269.33 & $\begin{array}{l}\text { LG (G } \\
141 C) \\
\end{array}$ & 3.10 & $\begin{array}{c}76.33 \\
(60.96) \\
\end{array}$ & 1133.33 & 2493.67 & $\begin{array}{l}19.50 \\
(4.40) \\
\end{array}$ & $\begin{array}{l}15.30 \\
(3.88) \\
\end{array}$ & $\begin{array}{c}8.20 \\
(2.77) \\
\end{array}$ & 19.33 \\
\hline Mean & 8.63 & 71.20 & 6.64 & 19.36 & 14.13 & 5.01 & 249.63 & - & 2.75 & $\begin{array}{c}70.89 \\
(57.63) \\
\end{array}$ & 1002.12 & 2250.03 & $\begin{array}{l}19.62 \\
(4.34) \\
\end{array}$ & $\begin{array}{l}15.20 \\
(3.79) \\
\end{array}$ & $\begin{array}{l}11.62 \\
(3.33) \\
\end{array}$ & 15.14 \\
\hline $\begin{array}{l}\text { S.E. (d) } \\
\pm\end{array}$ & 1.88 & 3.20 & 0.44 & 2.68 & 0.81 & 0.24 & 20.80 & - & 0.28 & 2.88 & 253.15 & 253.84 & 3.57 & 3.52 & 2.60 & 1.00 \\
\hline $\begin{array}{c}\text { C.D. }(0.0 \\
5)\end{array}$ & 3.76 & 6.40 & 0.88 & 5.36 & 1.61 & 0.48 & 41.65 & - & 0.55 & 3.83 & 506.80 & 508.18 & 0.88 & 1.04 & 0.83 & 2.01 \\
\hline
\end{tabular}

Where,

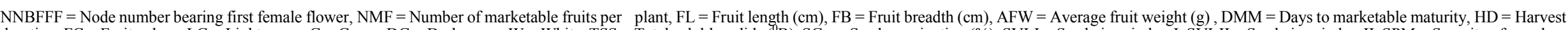

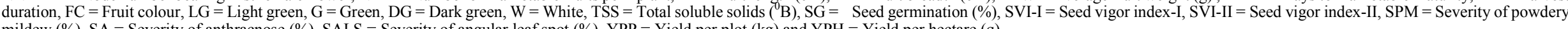
mildew $(\%), \mathrm{SA}=$ Severity of anthracnose $(\%)$, SALS $=$ Severity of angular leaf spot $(\%)$, YPP = Yield per plot $(\mathrm{kg})$ and $\mathrm{YPH}=$ Yield per hectare $(\mathrm{q})$

*Figures in the parenthesis are Arc sine transformed

** Figures in the parenthesis are Square root transformed 
Table 4. Estimates of phenotypic and genotypic coefficients of variability, heritability, genetic advance and genetic gain for different horticultural traits

\begin{tabular}{|c|c|c|c|c|c|c|c|}
\hline \multirow{2}{*}{ Characters } & \multicolumn{2}{|c|}{ Variance $\left(\sigma^{2}\right)$} & \multicolumn{2}{|c|}{ Coefficients of variability (\%) } & \multirow{2}{*}{$\begin{array}{c}\text { Heritability } \\
(\%)\end{array}$} & \multirow{2}{*}{$\begin{array}{l}\text { Genetic } \\
\text { advance }\end{array}$} & \multirow{2}{*}{$\begin{array}{c}\text { Genetic gair } \\
(\%)\end{array}$} \\
\hline & Phenotypic & Genotypic & Phenotypic & Genotypic & & & \\
\hline $\begin{array}{c}\text { Node number } \\
\text { bearing first }\end{array}$ & 11.39 & 6.10 & 39.12 & 28.63 & 53.60 & 3.73 & 43.22 \\
\hline $\begin{array}{c}\text { Days to } \\
\text { marketable }\end{array}$ & 49.94 & 34.58 & 9.93 & 8.26 & 69.20 & 10.07 & 14.14 \\
\hline $\begin{array}{l}\text { Number of } \\
\text { marketable }\end{array}$ & 1.42 & 1.13 & 18.00 & 16.06 & 79.60 & 1.96 & 29.52 \\
\hline $\begin{array}{c}\text { Harvest } \\
\text { duration (days) }\end{array}$ & 28.21 & 17.46 & 27.44 & 21.59 & 61.90 & 6.77 & 43.97 \\
\hline $\begin{array}{l}\text { Fruit length } \\
(\mathrm{cm})\end{array}$ & 9.34 & 8.36 & 21.63 & 20.47 & 89.60 & 5.64 & 39.92 \\
\hline $\begin{array}{l}\text { Fruit breadth } \\
(\mathrm{cm})\end{array}$ & 0.61 & 0.53 & 15.71 & 14.57 & 86.00 & 1.39 & 27.74 \\
\hline $\begin{array}{l}\text { Average fruit } \\
\text { weight }(\mathrm{g})\end{array}$ & 4605.67 & 3956.40 & 27.19 & 25.20 & 85.90 & 120.09 & 48.11 \\
\hline $\operatorname{TSS}\left({ }^{0} \mathrm{~B}\right)$ & 0.39 & 0.27 & 22.85 & 19.21 & 70.70 & 0.92 & 33.45 \\
\hline $\begin{array}{c}\text { Seed } \\
\text { germination }\end{array}$ & 75.99 & 63.53 & 12.30 & 11.24 & 83.60 & 15.01 & 21.17 \\
\hline $\begin{array}{c}\text { Seed vigor } \\
\text { index-I }\end{array}$ & 285685.49 & 189560.06 & 53.34 & 43.45 & 66.40 & 731.10 & 72.96 \\
\hline $\begin{array}{c}\text { Seed vigor } \\
\text { index-II }\end{array}$ & 292590.59 & 195941.54 & 24.04 & 19.67 & 67.00 & 746.57 & 33.18 \\
\hline $\begin{array}{c}\text { Severity of } \\
\text { powdery }\end{array}$ & 44.49 & 25.35 & 34.00 & 25.66 & 57.00 & 7.83 & 39.91 \\
\hline $\begin{array}{c}\text { Severity of } \\
\text { anthracnose }\end{array}$ & 33.39 & 14.77 & 38.02 & 25.29 & 44.00 & 5.24 & 34.47 \\
\hline $\begin{array}{c}\text { Severity of } \\
\text { angular leaf }\end{array}$ & 17.93 & 7.75 & 36.44 & 23.96 & 43.00 & 3.75 & 32.27 \\
\hline $\begin{array}{l}\text { Yield per plot } \\
(\mathrm{kg})\end{array}$ & 29.91 & 28.40 & 36.12 & 35.20 & 94.90 & 10.69 & 70.61 \\
\hline
\end{tabular}

\subsubsection{Parameters of Variability}

\subsubsection{Coefficients of Variability}

The estimates of phenotypic and genotypic coefficients of variability gave a clear picture of amount of variations presents in the available Germplasm (Table 4). For all the characters studied, phenotypic coefficients of variability were higher in magnitude than genotypic coefficients of variability, though difference was not much in all the cases. Thus, showing that these traits are not much influenced by environmental factors. Hence, selection based on phenotypic performance will be more reliable. Coefficients of variability varied in magnitude from character to character, indicating that there was a great diversity in the experimental material used. The genotypic coefficients of variability (GCV) were high for seed vigor index-I and yield per plot. This reflects greater genetic variability among the genotypes for these characters for making further improvement by selection. Whereas, moderate GCV were recorded for node number bearing first female flower, severity of powdery mildew, anthracnose and angular leaf spot, average fruit weight, harvest duration, fruit length, seed vigor index-II, total soluble solids, number of marketable fruits per plant and for fruit breadth. For, seed germination and days to marketable maturity, GCV were low. Similar results had also been reported by Singh (1997) and Yogesh et al. (2009).

\subsubsection{Heritability and Genetic gain}

The estimates of heritability were found high for the characters viz. yield per plot, fruit length and breadth, average fruit weight and seed germination and it was moderate for number of marketable fruits per plant, total soluble solids, days to marketable maturity, seed vigor index, harvest duration, severity of powdery mildew and node number bearing first female flower, while it was low for severity of anthracnose and angular leaf spot (Table 2). Kumar et al. (2008) reported high heritability estimates for fruit diameter, fruit length and fruit yield per plant and moderate heritability for node number bearing first female flower and days to fruit harvest. Similarly, high heritability was reported by Yogesh et al. (2009) for fruit diameter, fruit length, fruit weight at edible maturity and fruit yield per plant and moderate heritability was found for node number bearing first female flower. High heritability estimates for 
fruit length, fruit breadth, fruit weight and fruit yield per plant were also reported by Singh (1997) and Munshi et al. (2007). In the light of results obtained in the present studies, it is concluded that selection can be performed at phenotypic performance for highly heritable characters viz. fruit length, fruit breadth and average fruit weight and yield per plot.

Genetic gain (expressed as per cent of population mean) was low to high in nature for different characters. It was found high for seed vigour index-I and yield per plot. Moderate genetic gain was observed for average fruit weight, harvest duration, node number bearing first female flower, fruit length, severity of powdery mildew, anthracnose and angular leaf spot, total soluble solids, seed vigor index-II, number of marketable fruits per plant and fruit breadth, whereas it was recorded low for seed germination and days to marketable maturity. These findings are in line with Singh (1997), Kumar et al. (2008) and Yogesh et al. (2009).

High heritability estimates coupled with high genetic gain were observed for seed vigour index-I and yield per plot, indicated that these characters are under additive gene effects and these characters are more reliable for effective selection (Panse, 1957). Similar results for fruit yield per plant have been reported by Solanki and Seth (1980), Joshi et al. (1981), Rastogi and Rathore (1990) and Singh (1997). High heritability coupled with moderate genetic gain was observed for fruit length, fruit breadth and average fruit weight, indicated that these characters are under non-additive gene effects and selection for these characters will be less effective. Such traits are more under the influence of environment and do not respond to selection. Similar results were also reported by Joshi et al. (1981), Kumar et al. (2008) and Yogesh et al. (2009).

\subsection{Genetic Divergence Studies}

\subsubsection{Cluster composition}

After computing $\mathrm{D}^{2}$ values for all the possible pairs, 30 genotypes were grouped into 4 clusters, which indicated a large genetic diversity (Table 5). Maximum number of genotypes were accommodated in clusters-II (11) followed by cluster-III with 9 and cluster-I and cluster-IV with 5 genotypes, respectively. The resultant four clusters showed considerable genetic diversity. Genotypes from different geographical regions were grouped in the same cluster indicating no relationship between geographic distribution and genetic divergence, while genotypes collected from same location were grouped into different clusters, showing great genetic diversity. Similar results were also obtained by Rao et al. (2003), Khan (2006) and Kabir et al. (2009). Intra-cluster distance revealed that, cluster IV showed maximum intra-cluster distance (2.494) followed by cluster I (2.443), cluster II (2.410) and cluster III with minimum intra-cluster distance (2.383), respectively. Based on distance between clusters, i.e., inter-cluster distance, the maximum divergence was observed between cluster II and III (6.168) and lowest (3.432) was recorded between cluster I and III (Figure 1).

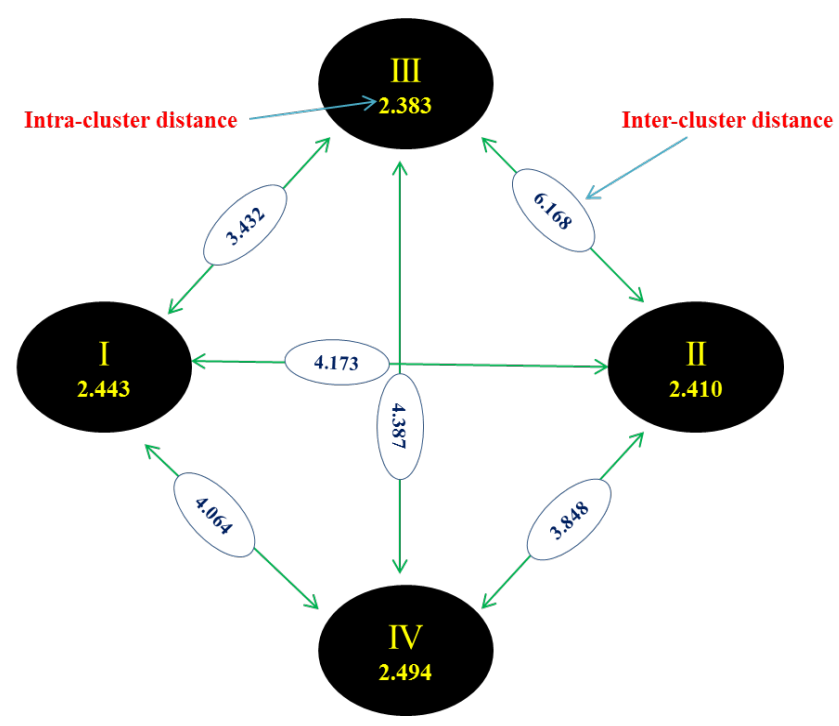

Figure 1. Diagram showing average intra and inter-cluster distances $\left(\mathrm{D}^{2}\right)$ of 30 genotypes of cucumberTable 5. Clustering pattern and average intra and inter cluster distance $\left(\mathrm{D}^{2}\right)$ of 30 genotypes of cucumber 
Table 5. Clustering pattern and average intra and inter cluster distance $\left(D^{2}\right)$ of 30 genotypes of cucumber

\begin{tabular}{|c|c|c|c|c|c|c|c|}
\hline Cluster & $\begin{array}{c}\text { Number of } \\
\text { genotvpes }\end{array}$ & Genotypes & Cluster & I & II & III & IV \\
\hline I & 5 & LC-6, LC-9, LC-19, LC-20, LC-26 & I & $\underline{2.443 *}$ & & & \\
\hline II & 11 & $\begin{array}{l}\text { LC-4, LC-7, LC-8, LC-10, LC-11, LC-13, } \\
\text { LC-16. LC-17. LC-18. LC-23. LC-24 }\end{array}$ & II & 4.173 & $\underline{2.410}$ & & \\
\hline III & 9 & $\begin{array}{c}\text { LC-1, LC-2, LC-3, LC-15, LC-21, LC-27, } \\
\text { LC-28. K-75. K-90 }\end{array}$ & III & 3.432 & 6.168 & $\underline{2.383}$ & \\
\hline IV & 5 & LC-5, LC-12, LC-14, LC-22, LC-25 & IV & 4.064 & 3.848 & 4.387 & 2.494 \\
\hline
\end{tabular}

* Diagonal bold figures represent the intra-cluster distance

Table 6. Cluster means for different characters among 30 genotypes of cucumber

\begin{tabular}{|c|c|c|c|c|}
\hline \multirow{2}{*}{ Characters } & \multicolumn{4}{|c|}{ Clusters } \\
\hline & I & II & III & IV \\
\hline Node number bearing first female flower & 9.20 & 10.77 & $6.34 *$ & 7.46 \\
\hline Number of marketable fruits per plant & 6.56 & 5.52 & 8.03 & 6.68 \\
\hline Fruit length $(\mathrm{cm})$ & 13.51 & 12.41 & 15.76 & 15.60 \\
\hline Fruit breadth $(\mathrm{cm})$ & 4.94 & 4.91 & 5.36 & 4.70 \\
\hline Average fruit weight $(\mathrm{g})$ & 245.33 & 212.12 & 299.15 & 247.33 \\
\hline Days to marketable maturity & 73.07 & 75.36 & 67.59 & 66.67 \\
\hline Harvest duration (days) & 17.93 & 15.27 & 25.52 & 18.67 \\
\hline TSS (0B) & 2.93 & 2.24 & 3.24 & 2.79 \\
\hline Seed germination (\%) & 80.80 & 63.64 & 76.26 & 67.27 \\
\hline Seed vigor index-I & 1453.67 & 559.73 & 1433.70 & 747.00 \\
\hline Seed vigor index-II & 2821.93 & 1799.00 & 2641.73 & 1965.31 \\
\hline Severity of powdery mildew (\%) & 20.42 & 23.90 & 14.84 & 18.02 \\
\hline Severity of anthracnose (\%) & 13.14 & 15.67 & 11.67 & 22.58 \\
\hline Severity of angular leaf spot (\%) & 12.56 & 13.80 & 10.42 & 8.04 \\
\hline Yield per plot (kg) & 14.36 & 10.54 & 21.38 & 14.79 \\
\hline
\end{tabular}

*Bold figures represent better performance of a character in the respective cluster

\subsubsection{Cluster means}

For crop improvement, inter-crossing among genotypes with outstanding mean performance was suggested by Roy and Sharma (1996). The cluster means of the various horticultural traits are presented in Table 6. Moreover, for getting the reliable conformity on the basis of cluster means, cluster-III exhibited higher means for number of marketable fruits per plant (8.03), fruit length (15.76), fruit breadth (5.36), average fruit weight (299.15), harvest duration (25.52), total soluble solids (3.24) and yield per plot (21.38) and lowest mean values for node number bearing first female flower (6.34) and severity of powdery mildew (14.84) and anthracnose (11.67). Cluster-I gave maximum mean values for seed germination (80.80) and seed vigour index-I (1453.67) and II (2821.93). Cluster-IV recorded minimum mean value for severity of angular leaf spot (8.04), while cluster-II did not possess superiority for any character. The genotypes having wide genetic base and desirable characteristics can be involved in intra-specific crosses which would lead to transmission of good genetic gain for various putative traits including yield for practical utility. Hence, Crossing between the genotypes of maximum two clusters (II and III) appeared to be most promising to combine the desirable characters. Earlier workers like Ram (2001), Kushwah et al. (2005) and Khan (2006) have also indicated the significance of genetic divergence. But, Mian and Bhal (1989) reported that parental clusters separated by medium $\mathrm{D}^{2}$ values had significant positive heterosis. Thus, heterosis could also be exploited by crossing between genotypes belonging to clusters with moderate diversity like genotypes of cluster I and II, cluster I \& IV and III \& IV. They are likely to produce new recombinant with desired traits.

Considering the magnitude of genetic distance and cluster means for different characters performance, the genotypes LC-22 and LC-25 of cluster IV could be selected for earliness and severity of angular leaf spot, and fruit 
length, respectively. In the cluster III the genotypes LC-1 for fruit breadth, fruit weight and total yield, LC-15 for number of fruits per plant and harvest duration, LC-28 for total soluble solids, LC-3 for seed vigor index-I and II, LC-15 for severity of anthracnose and LC-21 for severity of powdery mildew could be selected. The genotypes LC-6 and LC-20 of cluster I could be selected for better seed germination, if used in hybridization programme.

\section{REFERENCES}

[1] Abdul-Baki, A.A. and Anderson, J.D. 1973. Vigour germinated in soyabean seed by multiple criteria. Crop Sci. 13: 630-633.

[2] Allard, R.W. 1960. Principles of Plant Breeding. John Wiley and sons, New York. 485p.

[3] Anonymous. 1985. International rules for seed testing. Seed Sci. Tech. 13: 293-353.

[4] Anonymous. 2009. Package of practices for vegetable crops. Directorate of Extension Education, Dr. YS Parmar University of Horticulture and Forestry, Nauni, Solan. 202p.

[5] Bhat, N.A.; Masood, S.D.; Ahmad M. and Zargar M.R. 2007. Occurrence and severity of angular leaf spot of cucumber in Kashmir. Ann. Plant Protect. Sci. 15(2): 51-54.

[6] Burton, G.W. and DeVane, E.H. 1953. Estimating heritability in tall fescue (Festuca arundiancea) from replicated clonal material. Agron. J. 45: 478-481.

[7] Cohen, R.; Schreiber, S. and Nerson, H. 1995. Response of melofon breeding lines to powdery mildew, downy mildew, fusarium wilt and sudden wilt. Plant Dis. 79(6): 616-619.

[8] Das, S.; Maurya, K.R. and Choudhary D.N. 2003. Heritability studies in cucumber. J. App. Bio. 13(1\&2): 54-57.

[9] Gomez, K.A. and Gomez, A.A. 1983. Statistical Procedures for Agricultural Research. John Wiley and Sons Inc., New York. 680 p.

[10] Hamid, A.; Baloch, J. and Khan, N. 2002. Performance of six cucumber (Cucumis sativus L.) genotypes in Swat-Pakistan. Intl. J. Agric. Bio. 4(4): 491-492.

[11] Hanchinamani, C.N.; Patil, M. G.; Dharmatti, P.R. and Mokashi, A.N. 2008. Studies on variability in cucumber (Cucumis sativus L.). Crop Res. 36(1\&3): 273-276.

[12] Johanson, H.W.; Robinson, H.F. and Comstock, R.E. 1955. Estimates of genetic and environmental variability in soybean. Agron J. 47: 314-318.

[13] Joshi, S.; Joshi, M.C. and Vishnoi, A.K. 1981. Genotypic and phenotypic variability in cucumber. Vegetable Sci. 8(2): 114-119.

[14] Kabir, M.Y.; Khan, A.S.M.M.R. and Hassain, M.S. 2009. Genetic divergence in pointed gourd. J. Agric. Rural Dev. 7(1\&2): 87-92.

[15] Khan, A.S.M.M.R. 2006. Study on genetic diversity and production technology of pointed gourd. Ph.D. Thesis,
Bangladesh Agricultural University, Mymensingh, Bangladesh.

[16] Kumar, A. 2006. Studies on heterosis and inheritance of resistance to fruit fly in cucumber (Cucumis sativus L.). Ph.D. Thesis, Department of Vegetable Science, Dr. Y. S. Parmar University of Horticulture and Forestry, Nauni, Solan, H.P.

[17] Kumar, A.; Kumar, S. and Pal, A.K. 2008. Genetic variability and characters association for fruit yield and yield traits in cucumber. Indian J. Hort. 65(4): 423-428.

[18] Kushwah, S.; Bandyopadhya, B.B. and Sharma, R.N. 2005. Genetic divergence in brinjal. Haryana J. Hort. Sci. 34(3\&4): 316-317.

[19] Mahalanobis, P.C. 1936. On the generalized distance in statistics. Proceedings of National Academy of Science, India 2: 49-55.

[20] Mian, M.A.K. and Bhal, P.N. 1989. Genetic divergence and hybrid performance in chickpea. Indian J. Genet. 49(1): 119-129.

[21] Munshi, A.D.; Panda, B.; Behera, T.K.; Kumar, R.; Bisht, I. S. and Behera, T.K. 2007. Genetic variability in Cucumis sativus var. hardwickii R. germplasm. Cucurbit Genet. Coop. 30: 5-10.

[22] Nerson, H. 2007. Seed production and germinability of cucurbit crops. Seed Sci. Biotech. 1(1): 1-10.

[23] Panse, V.S. 1957. Genetics of quantitative characters in relation to plant breeding. Indian J. Genet. 17: 318-328.

[24] Ram, D. 2001. Non-Hirachical Euclidean Cluster analysis in pointed gourd. Indian J. Hort. 58(3): 264-268.

[25] Ransom, L.M.; Briens, R.G.O. and Glass, R.J. 1991. Chemical control of powdery mildew in green peas. Aus. Plant Patho. 20(1): 16-20.

[26] Rao, E.S.; Verma, V.K. and Munshi, A.D. 2003. Breeding potential of cucumber (Cucumis sativus L.) genotypes using D2 analysis. Indian J. Hort. 60(1): 53-58.

[27] Rao, R. 1952. Advanced statistical methods in biometrical research. John Wiley and Sons Inc., New York. 390p.

[28] Rastogi, K.B. and Rathore, A.D. 1990. A note on inter-relationship between yield and important plant characters of cucumber. Vegetable Sci. 17(1): 102-104.

[29] Roy, A. and Sharma, R.N. 1996. Multivariate analysis in chilli (Capsicum annum L.). Annals Agric. Res. 17(1): 30-32.

[30] Singh, M. 1997. Variability studies in some lines of cucumber (Cucumis sativus L.). M. Sc. Thesis, Department of Vegetable Science, Dr. Y. S. Parmar University of Horticulture and Forestry, Nauni, Solan, H.P.

[31] Singh, R.V.; Verma, T.S. and Thakur, P.C. 2002. Characters association in cucumber. Haryana J. Hort. Sci. 31(1\&2): 91-93.

[32] Solanki, S.S. and Seth, J.N. 1980. Correlation studies in cucumber (Cucumis sativus L.). Vegetable Sci. 7(2): 94-101.

[33] Tatlioglu, T. 1993. Cucumber (Cucumis sativus L.) p. 197-233. In: Genetic Improvement of Vegetable Crops. Kalloo, G. and B.O. Beorgh (eds.). Pergamon Press, Oxford. 
[34] Verma, S. 2003. Genetic variability and correlation studies in cucumber (Cucumis sativus L.). M. Sc. Thesis, Department of Vegetable Science, Dr. Y. S. Parmar University of Horticulture and Forestry, Nauni, Solan, H.P.

[35] Webner, T.C. and Shetty, N.V. 2000. Screening the cucumber germplasm collection for resistant to gummy stem blight in North Carolina field tests. Hort.Sci. 35(6): 1132-1140.

[36] Yawalkar, K.S. 1985. Vegetable Crops of India. Agri-Horticultural Publishing House, Nagpur.

[37] Yogesh, C.; Yadav, S.K.; Brijpal, B. and Dixit, S.K. 2009. Genetic variability, heritability and genetic advance for some traits in cucumber. Indian J. Hort. 66(4): 488-491. 